\title{
A Framework for Building an Interactive Satellite TV Based M-Learning Environment
}

\author{
$\underline{\text { doi:10.3991/ijim.v4i3.1295 }}$ \\ Ghassan F. Issa ${ }^{1}$, Shakir M. Hussain ${ }^{1}$ and Hussein Al-Bahadili ${ }^{2}$ \\ ${ }^{1}$ Petra University, Amman, Jordan \\ 2 The Arab Academy for Banking and Financial Sciences, Amman, Jordan
}

\begin{abstract}
This paper presents a description of an interactive satellite TV based mobile learning (STV-ML) framework, in which a satellite TV station is used as an integral part of a comprehensive interactive mobile learning (MLearning) environment. The proposed framework assists in building a reliable, efficient, and cost-effective environment to meet the growing demands of M-Learning all over the world, especially in developing countries. It utilizes recent advances in satellite reception, broadcasting technologies, and interactive TV to facilitate the delivery of gigantic learning materials. This paper also proposed a simple and flexible three-phase implementation methodology which includes construction of earth station, expansion of broadcasting channels, and developing true user interactivity. The proposed framework and implementation methodology ensure the construction of a true, reliable, and cost effective M-Learning system that can be used efficiently and effectively by a wide range of users and educational institutions to deliver ubiquitous learning.
\end{abstract}

Index Terms - E-Learning, Satellite TV, IPTV, E-Learning strategy, Interactive TV, Video On Demand (VoD).

\section{INTRODUCTION}

Eelectronic learning (E-Learning) is broadly defined as learning through electronic devices (e.g., mini-computers, desktop computers, CD players... etc.), which first emerged in the late 80s as a contender to classical face-toface learning. It is also referred to as distance learning (DLearning) [1]. In the 90s, due to (1) the impressive development in computer technologies, which leads to the emergent of powerful mobile laptop computers, (2) the remarkable technological advancement and expansion of wireless mobile communication systems, (3) emergent of advanced Internet technologies, and (4) the exponential growth in the processing power of wireless mobile devices while becoming more affordable and even ubiquitous due to the demand for business and social communications, a new form of learning was introduced. It was called mobile learning (M-Learning) [2, 3]. Figure 1 shows the functionality and mobility in the definition of M-Learning.

Since its emergent, a number of definitions have been given in the literature to M-Learning. One of the commonly used definitions for M-Learning is an evolved form of E-Learning that takes advantages of learning potential offered by the Internet and mobile devices technologies. It offers learning through mobile devices, which are also characterized by their limited-wireless connectivity, low-power battery and small-size, like mobile phones, smart phones, personal digital assistants (PDAs), Tablet PCs, e-Books, palmtops, and any other mobile microprocessor-based information technology devices that may be use in learning. These devices are also known as handhelds or portable devices [4].

There are a number of challenges that are limiting the wide-spread of reliable and cost-effective M-Learning which need to be carefully considered while developing M-Learning. These challenges can be categorized into technological and operation challenges [1, 5]. Technological challenges include: limited computing resources, limited wireless communication bandwidth, limited battery power, small screen size, and small keypad; while operational challenges may include: complexity, security, manageability, and unpredictability [6].

On the other hand, M-Learning requires handling and transmitting large amounts of text and multimedia materials, in addition to the demand of active participation of the learners. Such requirements place a heavy burden on internet service providers (ISP) who cannot supply their customers with proper bandwidth (low download rates) due to high Internet cost, and diverse and remote locations. This problem, of course, is clearly evident in many developing countries who still suffer from very low Internet penetration rates along with insufficient Internet bandwidth for multimedia contents, thus negatively affecting the basic requirements for an efficient M-Learning environment. A viable solution to overcome the shortcomings of the Internet and/or communication band-

\begin{tabular}{|c|c|c|c|c|}
\hline \multicolumn{2}{|c|}{ Functionality } & \multicolumn{3}{|c|}{ Mobility } \\
\hline $\begin{array}{c}\text { Desktop computers } \\
\text { Mini-computers } \\
\text { Workstations }\end{array}$ & Laptop computers & $\begin{array}{c}\text { PDAs } \\
\text { Palmtops }\end{array}$ & Smart phones & Mobile phones \\
\hline \multicolumn{2}{|c|}{ E-Learning } & \multicolumn{3}{|c|}{ M-Learning } \\
\hline
\end{tabular}

Figure 1. Functionality and mobility in the definition of M-Learning 
width would be to couple the satellite TV reception with broadband Internet. In such a case, satellite reception eliminates the major problem associated with low download rates, while Internet provides a mean for users' interactivity. In addition to that, the issue of low Internet penetration rate is solved since satellite reception has a much higher penetration rate.

The main objectives of this work are, first, to propose a framework for building a cost-effective and interactive MLearning environment by utilizing the widely spread satellite TV as a data download channel and Internet/telecommunication systems as a data upload channels. Second, revise an appropriate model for implementing the proposed framework in developing countries.

The rest of this paper is organized as follows: Section II provides an overview of existing related technologies, namely, Internet protocol TV (IPTV), video on demand (VoD), and network personal video recorder (NPVR). Section III describes the proposed framework and its main features. The procedure for user interaction is explained in Section IV. In Section V a description is given for one possible implementation methodology. Finally, in Section VI, some conclusions are drawn.

\section{OVERVIEW OF EXISTING TECHNOLOGIES}

In this section, we provide an overview on some important technologies that can play a big role in implementing our M-Learning framework.

\section{A. Internet Protocol TV (IPTV)}

The IP-based platform offers significant advantages such as: VoD, automatic archiving of the video broadcast in real media format, making interactive and personalized TV viewing, opportunity for integration and convergence, and generates statistics on programs/ channels/adverts viewed [9]. Therefore, such platform is referred to IPTV. Furthermore, IPTV can offer integration of voice, video, and data services for a wide-range of applications.

IPTV covers both live TV (multicasting) as well as stored video (VoD). The playback of IPTV requires either a personal computer or a set-top box (STB) connected to a TV. STB is a device that enables a TV set to become a user interface to the Internet and TV. It is the gateway to provide digital information to the home. It acts as a tuner for TV, controls access to many channels such as pay-perview, video gaming, ...., etc [10].

Video content is typically compressed using either MPEG-2 or MPEG-4 codec and then sent in an MPEG transport stream delivered via IP multicast in case of live TV or via IP unicast in case of VoD. IP multicast is a method in which information can be sent to multiple computers at the same time. The newly released MPEG-4 codec is increasingly used to replace the older MPEG-2.

The problem with IPTV, however, is the requirement of very high bandwidth internet connections, a commodity that is either unavailable or just too expensive in developing countries.

\section{B. Video on Demand (VoD)}

VoD permits a customer to browse an online program or film catalogue, to watch trailers and to then select a selected recording for playback. The playout of the selected movie starts nearly instantaneously on the customer's TV or PC.
Technically, when the customer selects the movie, a point-to-point unicast connection is set up between the customer's decoder (STBox or PC) and the delivering streaming server. The signaling for the trick play functionality (pause, slow-motion, wind/rewind etc.) is assured by real time streaming protocol (RTSP).

In an attempt to avoid content piracy, the VoD content is usually encrypted. Whilst encryption of satellite and cable TV broadcasts is an old practice, with IPTV technology it can effectively be thought of as a form of digital rights management. A film that is chosen, for example, may be playable for 24 hours following payment, after which time it becomes unavailable.

However, as we shall discuss in Section III, our proposed framework suggests a different form of VoD. It is based on downloading educational videos based on user's request and storing the video on the user's digital video recorder (DVR). The download takes place passively while the user is offline. The user can later view the downloaded video. While using this methodology (non-streaming) may appear, at first, old fashioned, it separates between downloading and viewing to overcome the limitations in the Internet speed.

\section{Network Personal Video Recorder (NPVR)}

The function of NPVR is similar to that of a DVR except it stores it content on a network instead of a local hard disk. This technology is convenient especially for educational institutions where users can access the recorded videos over a high speed local area network (LAN).

\section{THE PROPOSED FRAMEWORK}

This section presents a detail description of framework for building an interactive satellite TV based M-Learning (STV-ML) system. The framework consists of three main components. These are:

1- Centralized broadcasting center (CBC): To reduce the cost of satellite communications, and media production and editing, the proposed framework suggests the centralization of such facilities which can be later used on the basis of time sharing. The central facility consists of several components such as live and recorded broadcasting facility, earth station, a satellite channel connected to a well-known satellite, video servers, and video storage devices, web servers, SMS servers, and so forth.

2- Client side: Several clients (e.g., universities) can be connected to the CBC and can share the resources for reception and broadcasting of educational materials. The typical setting required by a university is minimal and consists of a satellite dish connected to a STB with DVR. It also requires a dedicated line of Internet connection (xDSL or leased line). If a university wishes, it can operate its own media production and editing units. A mobile broadcasting van can be readily made available for live broadcasting and live event coverage.

3- Communication and broadcasting channels: Three types of communication channels are required to provide broadcasting, reception, and interaction.

a) Interactive channel: This is a regular Internet connection between the user and the CBC. It consists of two channels: (i) Upload channel, which is used 
for uploading interactivity signals initiated by the user (online quires, requests, and responses); therefore, small bandwidth is fare enough to satisfy user needs. (ii) Download channel, which is a high speed connection used for passively downloading requested videos. In the case of home users, it is the same as the interactive channel which would be provided by an ADSL connection.

b) Satellite reception (client side): It is a satellite receiver or STB and a satellite dish through which a client can view live broadcasting.

c) Satellite broadcasting: Broadcasting can take place from the CBC using the dedicated earth station, or by using the mobile broadcasting stations (broadcasting van) located temporarily on the client side.

In the proposed framework, in order to overcome the drawback of the insufficient download bandwidth of current Internet and conventional telecommunication systems, a satellite TV communication channel is used for downloading bandwidth-demanding learning materials. On the other hand, to meet the demand of active participation of the learners, any standard Internet or telecommunication services can be utilized.

In order to build an interactive STV-ML system, first we need to establish a satellite broadcasting capabilities by constructing an earth station connected to one of the wellknown satellites (single channel). A broadcasting center and a production and editing centers are to be established and may well be integrated with a centralized M-Learning center managed by universities, educational institutions, training centers, etc. where they all can take advantages by producing and broadcasting educational materials. Universities can also rent air time to broadcast special advertisements about their institution, live camera feeds, meetings, conferences, and so on. Viewers can interact with programs using traditional approaches such as SMS messages, emails, and by phone. Figure 2 outlines the components of the proposed framework.

\section{A. Features of the proposed framework}

One of the main features of the proposed framework is its ability to overcome the limitations of Internet bandwidth while delivering E-Learning material for single and institutional users through the combination of satellite and Internet communications. Several other features and advantages may also include:

- Ability to deliver full-length high-quality multimedia educational programs

- Live coverage of teaching classes, local events, workshops, and international events

- M-Learning video broadcast

- Interactive TV capabilities

- Satellite video conferencing

- Important educational news coverage

- Interviews with prominent researchers and educators from all over the world

- Sharing of educational media across the country

- Educational media production

- Marketing locally produced educational material around the globe

- Marketing local universities internationally

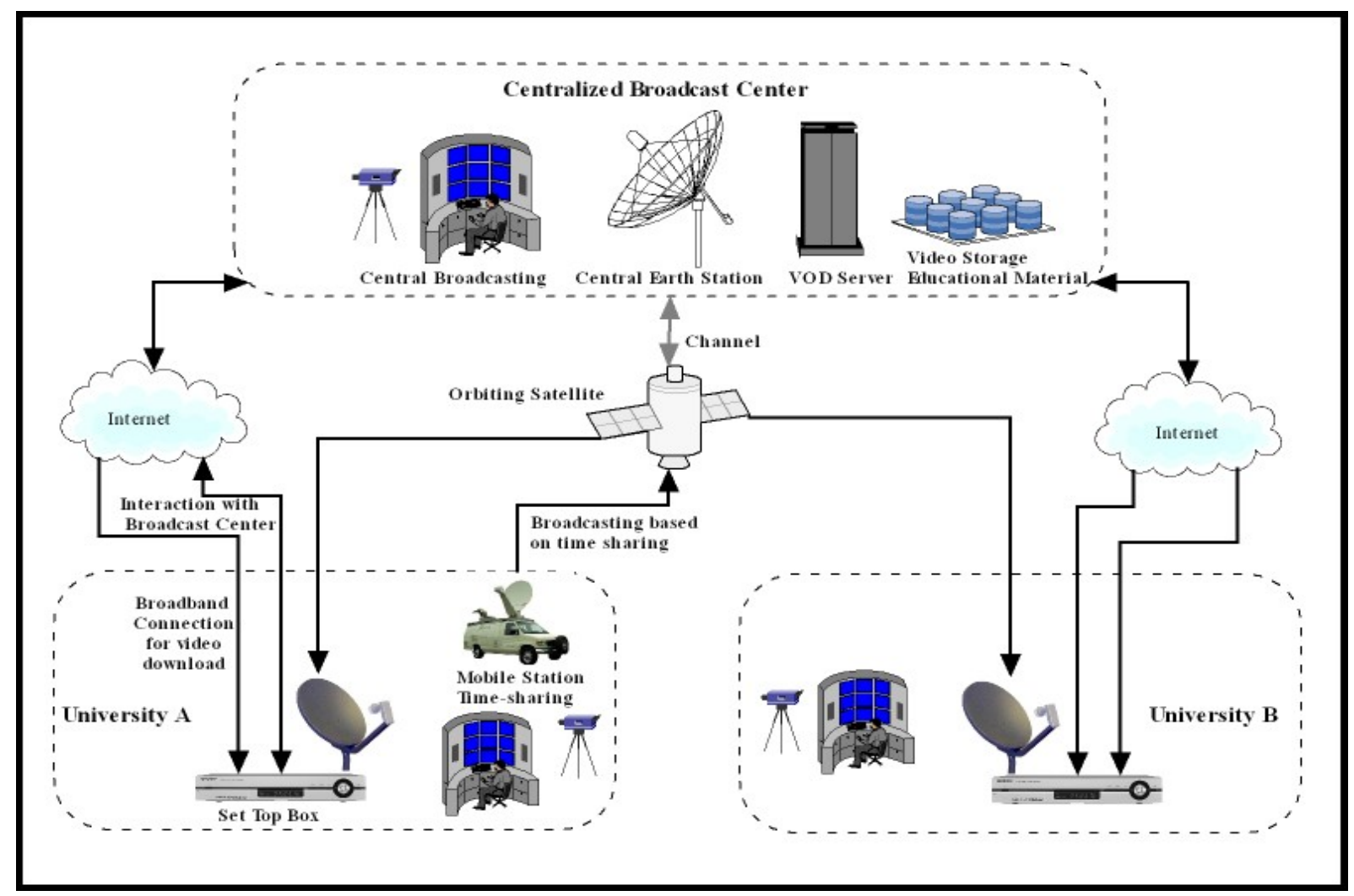

Figure 2. A CBC providing Universities with time-shared broadcasting and VoD services 
One of the other important feature of such learning system is that it can target a wide and different categories of users such as users who are looking for educational programs on TV who don't have or use PC and/or Internet access, too young or too old to use PC/Internet, unable to afford buying PC or subscribing for Internet, lacking motivation to use PC for learning. Those users are located at homes, schools, universities, and work. Furthermore, from user perspectives, current advances in satellite technologies make it more proper for delivering education materials than other IP-based technologies. This is because of the following:

- People are familiar with devices.

- People feel comfortable in using devices.

- Devices are available in their homes.

- Availability of interactive TV.

- Availability high speed Internet connections.

- Ability to control viewing of live TV (pause, forward, rewind).

- Ability to view a program while recording another.

- Ability to broadcast 3-D programs.

\section{USER INTERACTION}

User interaction is an important component and factor that needs to be carefully considered in designing and building a reliable STV-ML system. This is because it targets wide and different categories of users of different backgrounds. Fortunately, the enormous advancement in hardware and software technologies enable system developer to build a system the can meet all range of users, services, and applications.
Traditional TV is potentially interactive. For example, viewers compete mentally with quiz show participants, or co-operate between collocated groups. Moreover, viewers react emotionally to TV content, they record, edit and share TV content and discuss them either on/off time [7]. However, with the advancement in TV manufacturing technologies, interactive TV (ITV) technologies have been emerged. ITV describes a number of techniques that allow viewers to interact with TV content as they view it using one or more audiovisual and networked devices. In addition to technological aspects, the users experience different levels of interactivity defined ITV. ITV represents a continuum from low interactivity (TV on/off, volume, changing channels) to moderate interactivity (simple movies on demand without player controls) and high interactivity in which, for example, an audience member affects the program being watched [8]. Figure 3 shows the main components of a home-user interaction with broadcasting station and VoD services.

To be truly interactive, the viewer must be able to alter the viewing experience or return information to the broadcaster. This "return path" or "back channel" can be established by telephone, mobile SMS (text messages), radio, ADSL, or leased line.

Satellite TV viewers (mostly) return information to the broadcaster via their regular telephone lines. They are charged for this service on their regular telephone bill. An Internet connection via ADSL or other data communication technology is also being increasingly used.

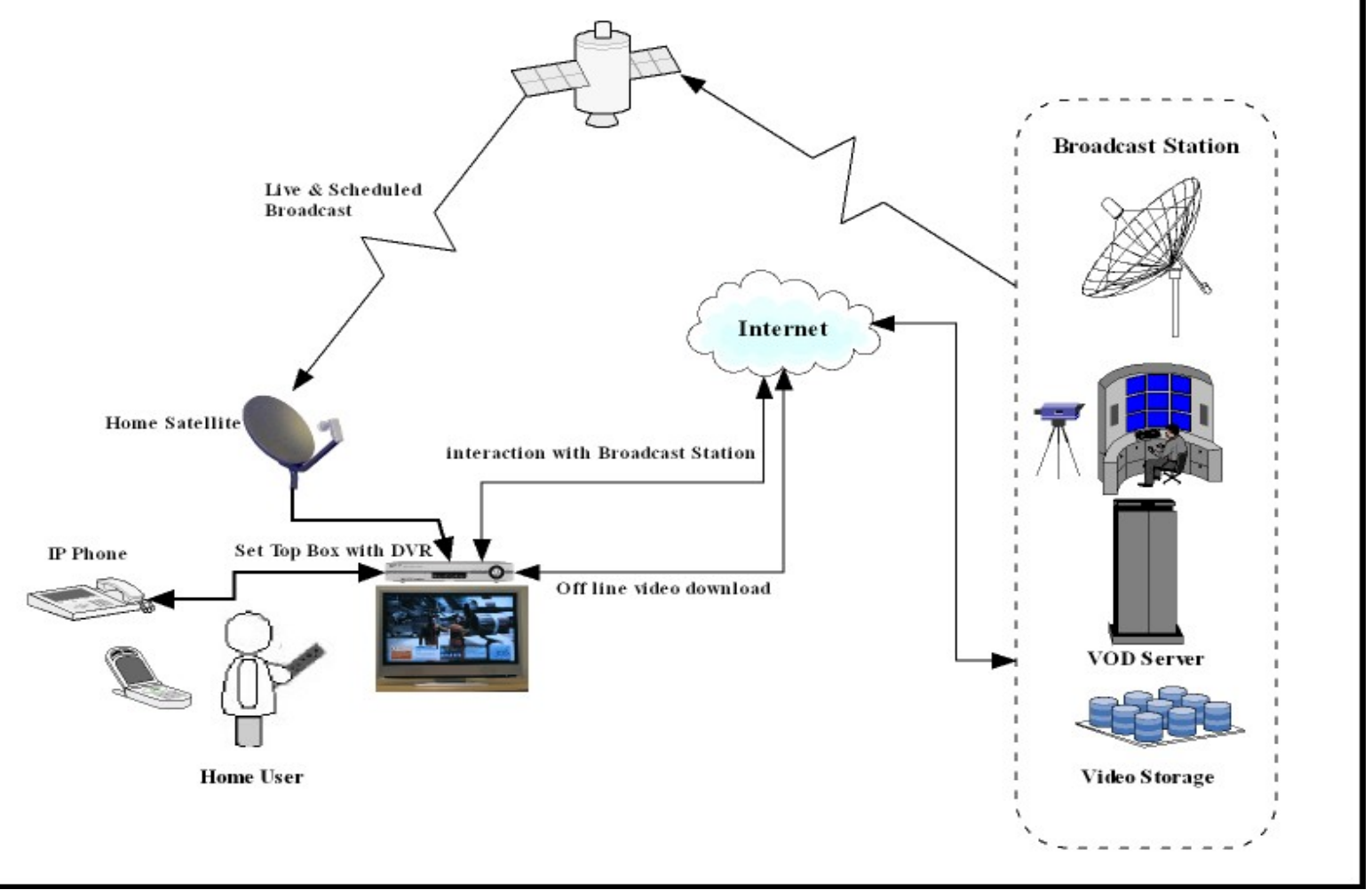

Figure 3. A home user with capabilities to interact with Broadcasting Station and video on demand Services 


\section{IMPLEMENTATION METHODOLOGY}

The STV-ML framework can be implemented to develop a reliable, efficient, and cost-effective education M-Learning system that can serve a wide range of users, especially in places characterize by:

- Low PC penetration rate due to affordability issues.

- Low Internet and broadband penetration.

- Bandwidth limitations (low throughput) could limit usage of advanced media; i.e., streaming video, large graphical and data files.

There are a number of methodologies that can be adopted in implementing our proposed framework which depends on the availability of the following factors:

1. Infrastructure.

2. Construction cost.

3. National and market needs.

4. Educational materials.

In this work, we propose a three-phase implementation methodology, these are:

1. Construct a single channel earth station.

2. Expand the number of channels.

Develop true M-Learning environment.

\section{Phase 1. Construct a single channel earth station}

In this phase, the basic transmit/receive earth station is constructed. It consists of the following major subsystems: antenna subsystem; low-noise amplifier subsystem; downlink subsystem; uplink subsystem; and earth station controller. In addition, this phase includes the construction of broadcast and media production/editing centers. A broadcasting center should be equipped with digital transmission components, networks and studios. The media production/editing center should be equipped with technologies to provide a complete solution for producing and editing of education materials. Other requirements may include: location of earth station, satellite connection, license, and space segment.

\section{Phase 2. Expand the number of channels}

In order to enhance the performance of the STV-ML system and to meet any future demands, we can expand the number of broadcast channels so that it can broadcast more than one education programs simultaneously. However, this may requires the expansion of the earth station reception capabilities.

\section{Phase 3. Introducing interactivity}

In this phase, a true M-Learning system can be developed by introducing high and flexible interactivity with the user. This can be achieved by using the advanced interaction technologies, such as IPTV, VoD, and NPVR, which are discussed in Section 2.

The main advantages of the three-phase implementation methodology are simplicity, low initial cost, cost effectiveness, and can be developed according to business, institutions, and users needs and demands.

\section{CONCLUSIONS}

In this paper, we presented a description and implementation methodology of an interactive STV-ML system. It utilizes satellite technologies to provide true M-Learning environment is a reliable, efficient, and cost effective way. Although, traditional TV broadcasting and IP-based MLearning is desirable in some cases, STV-ML is more convenient to many users who are located at home, schools, uni- versities, and work. This convenience comes as a result of the availability of satellite reception technologies and its usability, in addition to providing users with interactivity feature to interact with content broadcast centers. We have proposed a simple and flexible three-phase implementation methodology that can be implemented with low initial cost and expanded according to business, institutions, and user needs and demands.

\section{REFERENCES}

[1] Marguerite Leanne Koole. "The Framework for the Rational Analysis of Mobile Education (FRAME) Model: An Evaluation of Mobile Devices for Distance Education". Master thesis, Athabasca University, Alberta-Canada, 2006.

[2] Korneliya Yordanova. "Mobile Learning and Integration of Advanced Technologies in Education". International Conference on Computer Systems and Technologies, CompSysTech’07, 2007, pp. IV.23-1-6.

[3] Qing Li, Rynson W. H. Lau, Timothy K. Shih, Frederick W. Li. "Technology Supports for Distributed and Collaborative Learning over the Internet". ACM Transactions on Internet Technology, Vol. 8, No. 2, 2008. doi:10.1145/1323651.1323656

[4] Shahid Islam Wains and Waqar Mahmood, "Integrating M-Learning with E-Learning". Proceedings of the $9^{\text {th }}$ ACM Special Interest Group for Information Technology Education (SIGITE '08), pp. 31-37, Ohio-USA, October 16-18 2008.

[5] Rim Drira, Ichraf Tirellil, Mona Laroussi, Alain Derycke, Henda Benghezala, "What can we adapt in a Mobile Learning Systems?", Conference IMCL2006, Amman-Jordan, 19-21 April 2006.

[6] P. Paul Kroeker. "Technology to Facilitate Interaction in Mobile Learning". Master Thesis, Athabasca, Alberta, 2006.

[7] Chorianopolous, K. and Lekakos, G. 2007."Learn and Play with Interactive TV", ACM Comp. Entertain. Vol. 5, No 2, Article 3(August 2007), 9 pages. DOI = 10.145/1279540.1279544.

[8] Http://www.scn.ca/becomingasite.php, date: 4/11/08.

[9] The Arab Advisors Group Report, September, 2007, available at http://www.arabadvisors.com/Pressers/presser-200907.htm.

[10] Matej Zajc, Kemal Alic, Irena Battelino, Jurij Tasic, "Challenges of Interactive Digital Television for t-Learning", Zbornik Slovenske elektrotehniške konference ERK '07,September 2007.

\section{AUTHORS}

Ghassan F. Issa (gissa@uop.edu.jo). He received his B.E.T degree in Electronic Engineering from the University of Toledo, Ohio, in 1983, and B.S.EE in Computer Engineering from Tri-State University, Indiana in 1984. He received his M.S. and Ph.D. in Computer Science from Old Dominion University, Virginia, in 1987 and 1992 respectively. He was a faculty member and department chair of Computer Science at Pennsylvania College of technology (Penn State) from 1992 - 1995. He also served as faculty member and the dean of Computer Science at the Applied Science University in Amman, Jordan from 1995-2007. Currently he is an associate professor and the dean of Computer Science at Petra University in Amman, Jordan. His research interest covers block cipher, and authentication.

Shakir M. Hussain. He received his B.A. degree in statistics from University of Al-Mustansiriyah, Iraq, in 1976 and M.Sc. degrees in Computing and Information Science from Oklahoma State University, USA, in 1984. In 1997 he received the Ph.D. degree in Computer Science from University of Technology, Iraq. From 1997 to 2008 he was a faculty member at Applied Science University, Jordan. Currently, he is a head of Computer Science Department at Petra University, Jordan. His research interest covers block cipher, key generation, authentication, and data compression. He is a member of ACM.

Hussein Al-Bahadili received his B.Sc degree in Engineering from University of Baghdad in 1986. He received his 
M.Sc and $\mathrm{PhD}$ degrees in Engineering from University of London in 1988 and 1991, respectively. His field of study was parallel computers. He is currently associate professor at the Arab Academy for Banking \& Financial Sciences (AABFS). He is a visiting researcher at the Wireless Networks and Communications Centre (WNCC) at University of Brunel, UK. He is also a visiting researcher at the Centre of Osmosis Research and Applications (CORA), University of Surrey, UK. He has published many papers and book chapters in different fields of science and engineering in numer- ous leading scholarly and practitioner journals, and presented at leading world-level scholarly conferences. His research interests include parallel and distributed computing, wireless communications, computer networks, cryptography and network security, data compression, image processing, and artificial intelligence and expert systems.

Manuscript received on April $4^{\text {th }}$, 2010. Published as resubmitted by the authors on June $29^{\text {th }}, 2010$. 